Modified Bessel Functions of Half-Integral Order. The functions

$$
\begin{aligned}
& i_{n}=\sqrt{\pi / 2 x} I_{n+\frac{3}{3}}(x) \\
& k_{n}=(-1)^{n+1} \sqrt{\pi / 2 x} K_{n+\frac{1}{3}}(x)
\end{aligned}
$$

both satisfy the recurrence relation

$$
(2 p+1) z i_{p}=i_{p-1}-i_{p+1}
$$

and may be computed in a fashion entirely similar to the computation of the spherical Bessel functions. We have also

$$
\begin{gathered}
i_{0}=\frac{\sinh x}{x} \\
k_{0}=-\frac{\pi}{2 x} e^{-x} \\
k_{1}=\frac{\pi e^{-x}(x+1)}{2 x^{2}} .
\end{gathered}
$$

IRENE A. STEGUN

Milton AbRamowitz

National Bureau of Standards

Washington, D. C.

1. British Association for the Advancement of Science, Mathematical Tables, Volume $X$, Bessel Functions, Part II, Functions of Positive Integer Order, Cambridge, University Press, 1952.

2. L. Fox, $A$ Short Table for Bessel Functions of Integer Order and Large Arguments, Royal Society Shorter Mathematical Tables No. 3, Cambridge, 1954.

3. E. E. Allen, "Polynomial approximations to some modified Bessel functions," $M T A C$, v. 10,1956, p. $162-164$.

\title{
Remarks on the Disposition of Points in Numerical Integration Formulas
}

1. Introduction. Numerical integration formulas of degree 2 (i.e., exact for polynomials of at most degree 2) consisting of $n+1$ equally weighted points have been developed for certain regions in $n$-dimensional Euclidean space. Thacher [1] discusses the equations which a formula of this type must satisfy for regions which are invariant under the group of linear transformations which leave the $n$-cube with vertices $( \pm a, \pm a, \cdots, \pm a)$ invariant; we call these symmetric regions. Hammer and Stroud [2] give two such formulas for the $n$-simplex. Hammer [3] has shown that a set of $2 n$ equally weighted points lying on the coordinate axes form a formula of degree 3 for any symmetric region.

In section 2 of this note we show that the formulas of degree 2 discussed by Thacher can be described geometrically. We also show that there is a similar class of formulas for the regular $n$-simplex. In section 3 we describe geometrically a wide class of formulas of degree 3 containing $2 n$ points for symmetrical regions. 
2. Formulas of degree 2. This section is devoted to the proof of the following:

THEOREM 1. A necessary and sufficient condition that $n+1$ equally weighted points form a numerical integration formula of degree 2 for a symmetric region or for a regular n-simplex is that these points form the vertices of a regular $n$-simplex whose centroid coincides with the centroid of the region and lie on the surface of a sphere of radius $r=\sqrt{n I_{2} / I_{0}}$, where

$$
I_{0}=\int_{R} d v \quad I_{2}=\int_{R} x_{1}^{2} d v=\cdots=\int_{R} x_{n}^{2} d v .
$$

Proof. The weight for the points is $I_{0} /(n+1)$. We first prove the theorem for symmetrical regions; the proof for the $n$-simplex follows easily. Let $R$ be a symmetrical region. We then have

$$
\int_{R} x_{i} d v=\int_{R} x_{i} x_{j} d v=0 \quad i, j=1, \cdots, n \quad i \neq j .
$$

Now suppose the $n+1$ points

$$
\nu_{i}=\left(\nu_{i 1}, \nu_{i 2}, \cdots, \nu_{i n}\right) \quad i=0,1, \cdots, n
$$

are an integration formula of degree 2 for $R$ with equal weights. Then they satisfy the equations

$$
\text { (3) } \nu_{0 i} \nu_{0 j}+\nu_{1 i} \nu_{1 j}+\cdots+\nu_{n i} \nu_{n i}=\frac{(n+1) I_{2}}{I_{0}} \delta_{i j} \quad i, j=1, \cdots, n \text {. }
$$

To show that the points $\nu_{i}$ satisfy the conditions of the theorem consider the matrix

$$
A=\left[\begin{array}{ccccc}
\nu_{01} & \nu_{11} & \nu_{21} & \cdots & \nu_{n 1} \\
\nu_{02} & \nu_{12} & \nu_{22} & \cdots & \nu_{n 2} \\
\cdot & \cdot & \cdot & \cdot & \cdot \\
\nu_{0 n} & \nu_{1 n} & \nu_{2 n} & \cdots & \nu_{n n} \\
\sqrt{I_{2} / I_{0}} & \sqrt{I_{2} / I_{0}} & \sqrt{I_{2} / I_{0}} & \cdots & \sqrt{I_{2} / I_{0}}
\end{array}\right]
$$

By (2) and (3) it is seen that

where $I$ is the identity matrix. Hence

$$
A A^{T}=\frac{(n+1) I_{2}}{I_{0}} I
$$

$$
A^{T} A=\frac{(n+1) I_{2}}{I_{0}} I
$$

is equivalent to the equations

(4) $\nu_{i 1} \nu_{j 1}+\cdots+\nu_{i n} \nu_{j n}+\frac{I_{2}}{I_{0}}=\frac{(n+1) I_{2}}{I_{0}} \delta_{i j} \quad i, j=0,1, \cdots, n$.

This shows that the points lie on the sphere of radius $\sqrt{n I_{2} / I_{0}}$ with centroid at the origin. To show that the $\nu_{i}$ are vertices of a regular $n$-simplex it suffices to 
show that they are equidistant. By (4)

$$
\begin{array}{r}
d^{2}\left(\nu_{i}, \nu_{j}\right)=\nu_{i 1}^{2}+\cdots+\nu_{i n}^{2}+\nu_{j 1}^{2}+\cdots+\nu_{j n}^{2}-2\left(\nu_{i 1} \nu_{j 1}+\cdots+\nu_{i n} \nu_{j n}\right) \\
=\frac{2(n+1) I_{2}}{I_{0}} .
\end{array}
$$

Reversal of the above argument proves the condition of the theorem is sufficient. This establishes the theorem for symmetric regions.

To show the theorem holds for the $n$-simplex consider a particular set of $n+1$ equidistant points which lie on an $n$-sphere with center at the origin and radius $r=a \sqrt{1 /(n+2)}$. The results in [2] show that there exists a regular $n$-simplex $S_{n}$ for which these points are an integration formula. The vertices of $S_{n}$ lie on a sphere of radius $a$. The above argument shows that any $n+1$ equidistant points on the sphere of radius $r$ is also an integration formula for $S_{n}$. This completes the proof of the theorem.

\section{TABLE 1}

$\begin{array}{cc}\text { Region } & I_{2} / I_{0} \\ P_{n} & a^{2} /(n+2) \\ S_{n} & a^{2} / n(n+2) \\ C_{n} & a^{2} / 3 \\ Q_{n} & 2 a^{2} /(n+1)(n+2)\end{array}$

Values of $I_{2} / I_{0}$ are given in Table 1 for four regions (i.e., their interiors). $P_{n}$ is the $n$-sphere of radius $a$ with centroid at the origin. $S_{n}$ is any regular $n$ simplex whose vertices lie on $P_{n}$. $C_{n}$ is the $n$-cube with vertices $( \pm a, \pm a, \cdots, \pm a)$ or this cube rotated in any manner. $Q_{n}$ is the region defined by the $2^{n}$ inequalities $\pm x_{1} \pm x_{2} \pm \cdots \pm x_{n} \leq a$, or any rotation of this region. The regions $S_{n}, C_{n}$, and $Q_{n}$ are the only regular polytopes for $n \geq 5$ ([4], p. 120). In the following section we use the fact that any vertex of $Q_{n}$ is a distance of $a \sqrt{2}$ from $2(n-1)$ vertices and a distance of $2 a$ from one vertex.

3. Formulas of degree 3 . In this section we prove

TheOREM 2. A necessary and sufficient condition that $2 n$ points $\nu_{1}, \cdots, \nu_{n}$, $-\nu_{1}, \cdots,-\nu_{n}$ form an equally weighted numerical integration formula of degree 3 for a symmetrical region is that these points form the vertices of a $Q_{n}$ whose controid coincides with the centroid of the region and lie on an $n$-sphere of radius $r=\sqrt{n I_{2} / I_{0}}$.

Proof. The weight is $\frac{1}{2 n} I_{0}$. Let the points
$\nu_{i}=\left(\nu_{i 1}, \cdots, \nu_{i n}\right)$
$-\nu_{i}=\left(-\nu_{i 1}, \cdots,-\nu_{i n}\right)$
$i=1, \cdots, n$

satisfy

$$
\nu_{1 i} \nu_{1 j}+\cdots+\nu_{n i} \nu_{n j}=\frac{n I_{2}}{I_{0}} \delta_{i j} \quad i, j=1, \cdots, n \text {. }
$$


(The integrals of the odd degree monomials are zero and are identically satisfied.) Setting

$$
A=\left[\begin{array}{cccc}
\nu_{11} & \nu_{21} & \ldots & \nu_{n 1} \\
\nu_{12} & \nu_{22} & \cdots & \nu_{n 2} \\
\cdot & \cdot & \cdot & \cdot \\
\nu_{1 n} & \nu_{2 n} & \cdots & \nu_{n n}
\end{array}\right]
$$

the proof follows as in theorem 1. We find

$$
d^{2}\left( \pm \nu_{i}, \pm \nu_{j}\right)=\frac{2 n I_{2}}{I_{0}} \quad i \neq j \quad i, j=1, \cdots, n
$$

and

$$
d^{2}\left(\nu_{i},-\nu_{i}\right)=\frac{4 n I_{2}}{I_{0}}
$$

This completes the proof.

The results of theorems 1 and 2 generalize results given by Thacher for the 2-cube (square) and the 3-cube. There may exist unequally weighted integration formulas containing fewer points than those discussed here; however, the equally weighted formulas will probably remain important for applications.

4. Concluding remarks. One of the properties desirable in an integration formula is that the points be interior to the region. The $2 n$ point formula given by Tyler [5] for $C_{n}$ using points on the coordinate axes has the points outside for $n>3$. We give here two formulas for $C_{n}$ (with vertices $( \pm 1, \pm 1, \cdots, \pm 1)$ ) of degrees 2 and 3 for which the points are interior for all $n([4], 245)$ :

Let $\Gamma_{k}$ denote the point $\left(\gamma_{1}, \gamma_{2}, \cdots, \gamma_{n}\right)$ where

$$
\begin{aligned}
& \gamma_{2 r-1}=\sqrt{\frac{2}{3}} \cos \frac{2 r k \pi}{n+1} \quad \gamma_{2 r}=\sqrt{\frac{2}{3}} \sin \frac{2 r k \pi}{n+1} \\
& r=1,2, \cdots,\left[\frac{1}{2} n\right]
\end{aligned}
$$

( $\left[\frac{1}{2} n\right]$ is the greatest integer not exceeding $\left.\frac{1}{2} n\right)$, and if $n$ is odd $\gamma_{n}=(-1)^{k} / \sqrt{3}$. Then $\Gamma_{0}, \Gamma_{1}, \cdots, \Gamma_{n}$ satisfy the conditions of theorem 1 , and all are interior to $C_{n}$.

Let $\Sigma_{k}$ denote the point $\left(\sigma_{1}, \sigma_{2}, \cdots, \sigma_{n}\right)$ where

$$
\begin{aligned}
\sigma_{2 r-1}=\sqrt{\frac{2}{3} \cos \frac{(2 r-1) k \pi}{n} \quad \sigma_{2 r}=\sqrt{\frac{2}{3} \sin \frac{(2 r-1) k \pi}{n}}}, \\
r=1,2, \cdots,\left[\frac{1}{2} n\right]^{\prime}
\end{aligned}
$$

and if $n$ is odd $\sigma_{n}=(-1)^{k} / \sqrt{3}$. Then $\Sigma_{1}, \cdots, \Sigma_{2 n}$ satisfy the conditions of theorem 2 and all are interior to $C_{n}$.

For the formulas and regions we have considered, only the location of the points relative to each other and not to the region was found to be important. The insight given by these results may prove useful in further investigations; one problem is that concerning formulas for more general regions.

University of Wisconsin

A. H. Stroud Madison, Wisconsin

This work was supported in part by the Office of Ordnance Research, U. S. Army Contract No. DA-11-022-ORD-2301, and in part by the Wisconsin Alumni Research Foundation. 
1. Henry C. Thacher, Jr., "Optimum quadrature formulas in $s$ dimensions," $M T A C$, v. 11, 1957, p. 189-194.

2. Preston C. Hammer \& Arthur H. Stroud, "Numerical integration over simplexes," $M T A C$, v. 10,1956, p. $137-139$.

3. P. C. HAMmer, Private communication.

4. H. S. M. CoXeter, Regular Polytopes, Methuen \& Co. Ltd., London, 1948.

5. G. W. TyLER, "Numerical integration of functions of several variables," Canadian Jn. Math., v. 5, 1953, p. 393-412.

\section{TECHNICAL NOTES AND SHORT PAPERS}

\section{A Method for the Numerical Evaluation of Certain Infinite Integrals}

The solution of many physical problems often necessitates the numerical evaluation of infinite real integrals, a common example being that of solutions obtained with the aid of integral transforms. The evaluation of such integrals is often a laborious task, particularly if the integrand is oscillatory, so that it is usual to resort to special methods which give information for certain ranges of values of the variables; methods of this type are those involving asymptotic expansions or the related techniques of steepest descent and of stationary phase. The purpose of the present note is to outline a method in which the value of such integrals is expressed in terms of a convergent series obtained by a modification of the corresponding asymptotic expansion. The development is given below for a special case only, namely one which might arise in conjunction with the use of sine transforms; it will be clear however that these results can be readily generalized to other types of integrals which are usually reduced to an asymptotic representation. Examples may be found in Erdélyi [1]. The method is thus valid whether the integrand is oscillatory or not; in fact, though the special integrand considered in detail below does oscillate, inspection of the convergence proofs shows that this fact is of little importance to the developments presented. A method which holds in the case of oscillatory integrands has been described by I. M. Longman [2].

Basic expansions. Consider a convergent integral $I(a)$ of the form

$$
I(a)=\int_{a}^{\infty} f(x) \sin x d x ; \quad f(x) \rightarrow 0 \text { steadily as } x \rightarrow \infty .
$$

By $f(x) \rightarrow 0$ steadily, we mean that $f\left(x_{1}\right) \geq f\left(x_{2}\right)>0$ if $x_{1}<x_{2}$ and $\lim f(x)=$ 0 ; see Whittaker and Watson [3]. $N$ successive integrations by parts may be shown to give the following result

$$
I(a)=\sum_{i=0}^{N} f^{(i)}(a) \cos [a+i(\pi / 2)]+\int_{a}^{\infty} f^{(N)}(x) \sin [x+N(\pi / 2)] d x
$$

where $f^{(i)}=\left(d^{i} f / d x^{i}\right)$, provided that $f(x)$ is differentiable the required number of times, and that

$$
f^{(i)}(x) \rightarrow 0 \text { steadily as } x \rightarrow \infty ; \quad i=0,1,2, \cdots .
$$

The term in equation (2) containing the summation usually represents an asymptotic representation of $I$ for large values of $a$, and the infinite series obtained as $N$ is increased indefinitely in general does not converge. A convergent expansion 